\title{
FACTOR GENERALIZED BE-SEMIGROUPS THROUGH DEDUCTIVE SYSTEMS
}

\author{
S. SALEEM ${ }^{1}$, F. HUSSAIN ${ }^{1 *}$, B. DAVVAZ ${ }^{2}$ and M.T. RAHIM ${ }^{3}$ \\ ${ }^{1,3}$ Department of Mathematics, Abbottabad University of Science and Technology, Pakistan. \\ ${ }^{2}$ Department of Mathematics Yazd University, Yazd, Iran \\ *Corresponding author. E-mail: fawad.hussain30@gmail.com
}

\section{DOI:10.20948/mathmontis-2021-51-4}

Summary. In this paper, we investigate some properties of homomorphisms and deductive systems of generalized BE-semigroups. In particular, we show that through every deductive system, we may get factor generalized BE-semigroup.

\section{INTRODUCTION}

Is'eki and Tanaka defined two classes of abstract algebras, i.e. BCK-algebras and BCI-algebras [1, 2] while $\mathrm{Hu}$ and $\mathrm{Li}$ also defined two classes of abstract algebras, i.e. $\mathrm{BCH}$-algebra and proper $\mathrm{BCH}$-algebra [3, 4]. It is well known that every BCK-algebra is a BCI-algebra, i.e. a BCI-algebra is a generalization of a BCK-algebra. The authors of [3] have shown that every BCI-algebra is a BCH-algebra. For general information on BCK-algebras, we refer to [5]. Neggers and Kim [6] introduced the concept of a d-algebra which is a generalization of BCK-algebras, and also they introduced the concept of a B-algebra [7, 8], that is, (i) $x * x=1$, (ii) $x * 1=x$, (iii) $(x * y) * z=x *(z *(1 * y))$, for any $x, y, z \in X$, which is equivalent to the idea of groups. Furthermore, Jun et al. [9] introduced a new concept, known as an BH-algebra, which is another generalization of BCH/BCI/BCK-algebras, that is, (i), (ii), and (iii) $x * y=1$ and $y * x=1$ imply that $x=y$ for any $x, y \in X$. Walendziak obtained some equivalent set of axioms for a B-algebra [10]. Kim et al. [11] introduced the concept of a (pre-) Coxeter algebra and showed that a Coxeter algebra is equivalent to an abelian group all of whose elements have order 2, that is, a Boolean group. C. B. Kim and H. S. Kim [12] introduced the concept of a BM-algebra which is a specialization of B-algebras. They proved that the class of BM-algebras is a proper subclass of B-algebras and also showed that a BM-algebra is equivalent to a 1-commutative B-algebra. In [13], H. S. Kim and Y. H. Kim introduced the concept of a BE-algebra as a generalization of a BCK-algebra. Using the concept of upper sets, they gave an equivalent condition of the filters in BE-algebras. In $[14,15]$, Ahn and So introduced the concept of ideals, upper sets as well as generalized upper sets in BE-algebras and obtained several properties of the said concepts. In [16], by combining BE-algebra and semigroup, the authors introduced the concept of a BE-semigroup and explored some elegant and interesting properties. Recently in [17], the authors have given a new generalization of a BE-algebra which is called a PSRU-algebra. They have defined left (resp. right) ideal as well as filter in the said structure and discussed a relationship between left ideal and filter.

2010 Mathematics Subject Classification: 06F35 (20M99).

Keywords and Phrases: GBE-semigroup, Congruence relation, Homomorphism, Deductive system. 


\section{PRELIMINARIES}

In this section, we discuss the structure of a generalized BE-semigroup which is a generalization of a BE-semigroup. We give some examples and characterize it by some properties. Moreover, we discuss some classes of generalized BE-semigroups. We start from the definition of a generalized BE-algebra and this definition has been taken from the source [18].

\section{Definition 2.1}

Let $\boldsymbol{T}$ be a non-empty set equipped with a binary operation "*" and a constant " $1_{\boldsymbol{T}}$ ", then it is called a generalized BE-algebra (denoted by GBE-algebra) if the following conditions hold:

(i) $\forall u \in T, u * u=1_{T}$,

(ii) $\forall u \in \boldsymbol{T}, u * 1_{T}=1_{T}$,

(iii) $\forall u, v, w \in \boldsymbol{T}, u *(v * w)=v *(u * w)$.

The following example shows that the above structure exists.

\section{Example 2.2 [18]}

Assume that $\boldsymbol{K}=\left\{1_{\boldsymbol{K}}, r, t\right\}$ and " $*$ " is defined in $\boldsymbol{K}$ in the table given below.

\begin{tabular}{|c|c|c|c|}
\hline$*$ & $1_{\boldsymbol{K}}$ & $r$ & $t$ \\
\hline $1_{\boldsymbol{K}}$ & $1_{\boldsymbol{K}}$ & $1_{\boldsymbol{K}}$ & $1_{\boldsymbol{K}}$ \\
\hline$r$ & $1_{\boldsymbol{K}}$ & $1_{\boldsymbol{K}}$ & $t$ \\
\hline$t$ & $1_{\boldsymbol{K}}$ & $r$ & $1_{\boldsymbol{K}}$ \\
\hline
\end{tabular}

Then one can easily show that $\left(\boldsymbol{K} ; *, 1_{\boldsymbol{K}}\right)$ is a GBE- algebra.

It should be noted that in GBE-algebra $\left(\boldsymbol{L} ; *, 1_{\boldsymbol{L}}\right)$, we define a relation " $\leq$ " by $m \leq n \Leftrightarrow$ $m * n=1_{L}$. Moreover, in this paper, we shall assume that $m \leq n$ and $n \leq m$ always implies that $m=n$. Now it can easily be seen that the below properties hold in a GBE-algebra $\left(\boldsymbol{L} ; *, 1_{\boldsymbol{L}}\right)$.

(i) $m *(n * m)=1_{L}$,

(ii) $m *((m * n) * n)=1_{L}$.

Moreover, we have the definition given below which has been taken from [18].

\section{Definition 2.3}

A GBE-algebra $\left(\boldsymbol{R} ; *, 1_{\boldsymbol{R}}\right)$ is called self-distributive if

$$
u *(v * w)=(u * v) *(u * w) \forall u, v, w \in \boldsymbol{R} .
$$


We now give an example to show the existence of a self-distributive GBE-algebra.

\section{Example 2.4 [18]}

Let $\boldsymbol{D}=\left\{1_{\boldsymbol{D}}, c, d\right\}$ and “*” is defined in $\boldsymbol{D}$ in the table given below:

\begin{tabular}{|c|c|c|c|}
\hline$*$ & $1_{\boldsymbol{D}}$ & $c$ & $d$ \\
\hline $1_{\boldsymbol{D}}$ & $1_{\boldsymbol{D}}$ & $1_{\boldsymbol{D}}$ & $1_{\boldsymbol{D}}$ \\
\hline$c$ & $1_{\boldsymbol{D}}$ & $1_{\boldsymbol{D}}$ & $d$ \\
\hline$d$ & $1_{\boldsymbol{D}}$ & $c$ & $1_{\boldsymbol{D}}$ \\
\hline
\end{tabular}

Then it is easy to check that $\boldsymbol{D}$ is a self-distributive GBE-algebra.

Furthermore, we have the definitions given below which have been taken from [18].

\section{Definition 2.5}

Let $(\boldsymbol{A}, *)$ and $(\boldsymbol{B}, *)$ be two GBE-algebras. A mapping $\varphi: \boldsymbol{A} \rightarrow \boldsymbol{B}$ is called a homomorphism from $\boldsymbol{A}$ into $\boldsymbol{B}$ if

$$
\varphi(c * d)=\varphi(c) * \varphi(d)
$$

holds $\forall c, d \in A$.

\section{Definition 2.6}

Let $\emptyset \neq \boldsymbol{H}$ be a set which is equipped with two binary operations " $\odot$ " and "*" and a constant " $1_{\boldsymbol{H}}$ ", then $\boldsymbol{H}$ is called a GBE-semigroup if the following conditions are satisfied:

(i) $(\boldsymbol{H} ; \odot)$ is a semigroup,

(ii) $\left(\boldsymbol{H} ; *, 1_{\boldsymbol{H}}\right)$ is a GBE-algebra,

(iii) “ $\odot$ ” is distributive over “*”, i.e.,

$$
u \odot(v * w)=(u \odot v) *(u \odot w) \text { and }(u * v) \odot w=(u \odot w) *(v \odot w) \forall u, v, w \in \boldsymbol{H} .
$$

Let us give some examples in order to understand the above structure.

\section{Example 2.7 [18]}

Assume that $\boldsymbol{L}=\left\{1_{\boldsymbol{L}}, e, f, \mathrm{~g}\right\}$ and define " $\odot$ " and "*" in $\boldsymbol{L}$ in the tables given below: 


\begin{tabular}{|c|c|c|c|c|}
\hline$\odot$ & $1_{L}$ & $e$ & $f$ & $g$ \\
\hline $1_{L}$ & $1_{L}$ & $1_{L}$ & $1_{L}$ & $1_{L}$ \\
\hline$e$ & $1_{L}$ & $1_{L}$ & $1_{L}$ & $1_{L}$ \\
\hline$f$ & $1_{L}$ & $1_{L}$ & $1_{L}$ & $e$ \\
\hline$g$ & $1_{L}$ & $1_{L}$ & $1_{L}$ & $1_{L}$ \\
\hline
\end{tabular}

\begin{tabular}{|c|c|c|c|c|}
\hline$*$ & $1_{L}$ & $e$ & $f$ & $g$ \\
\hline $1_{L}$ & $1_{L}$ & $1_{L}$ & $1_{L}$ & $1_{L}$ \\
\hline$e$ & $1_{L}$ & $1_{L}$ & $1_{L}$ & $1_{L}$ \\
\hline$f$ & $1_{L}$ & $e$ & $1_{L}$ & $e$ \\
\hline$g$ & $1_{L}$ & $1_{L}$ & $1_{L}$ & $1_{L}$ \\
\hline
\end{tabular}

Then one can easily show that $\left(\boldsymbol{L} ; \odot, *, 1_{\boldsymbol{L}}\right)$ is a GBE-semigroup.

\section{Example 2.8 [18]}

Assume that $\boldsymbol{W}=\left\{1_{W}, a, b, c, d\right\}$ and define " $\odot$ "and " $*$ " in $\boldsymbol{W}$ in the tables given below:

\begin{tabular}{|c|c|c|c|c|c|}
\hline$\odot$ & $1_{W}$ & $a$ & $b$ & $c$ & $d$ \\
\hline $1_{W}$ & $1_{W}$ & $1_{W}$ & $1_{W}$ & $1_{W}$ & $1_{W}$ \\
\hline$a$ & $1_{W}$ & $1_{W}$ & $1_{W}$ & $1_{W}$ & $1_{W}$ \\
\hline$b$ & $1_{W}$ & $1_{W}$ & $1_{W}$ & $1_{W}$ & $1_{W}$ \\
\hline$c$ & $1_{W}$ & $1_{W}$ & $1_{W}$ & $1_{W}$ & $1_{W}$ \\
\hline$d$ & $1_{W}$ & $1_{W}$ & $1_{W}$ & $1_{W}$ & $d$ \\
\hline
\end{tabular}

\begin{tabular}{|c|c|c|c|c|c|}
\hline$*$ & $1_{W}$ & $a$ & $b$ & $c$ & $d$ \\
\hline $1_{W}$ & $1_{W}$ & $b$ & $b$ & $c$ & $1_{W}$ \\
\hline$a$ & $1_{W}$ & $1_{W}$ & $1_{W}$ & $c$ & $1_{W}$ \\
\hline$b$ & $1_{W}$ & $1_{W}$ & $1_{W}$ & $1_{W}$ & $d$ \\
\hline$c$ & $1_{W}$ & $a$ & $b$ & $1_{W}$ & $1_{W}$ \\
\hline$d$ & $1_{W}$ & $1_{W}$ & $1_{W}$ & $1_{W}$ & $1_{W}$ \\
\hline
\end{tabular}

Then it is obvious that $\left(\boldsymbol{W} ; \odot, *, 1_{W}\right)$ is a GBE-semigroup.

Let us state some properties.

Proposition 2.9 [18]

The below properties are true for a GBE-semigroup $\left(\boldsymbol{M} ; \odot, *, 1_{\boldsymbol{M}}\right)$.

(i) $1_{M} \odot u=u \odot 1_{M}=1_{M} \forall u \in \boldsymbol{M}$,

(ii) $u \leq v \Rightarrow u \odot w \leq v \odot w, w \odot u \leq w \odot v \forall u, v, w \in \boldsymbol{M}$. 
We are now going to define deductive system. For the definition given below we refer the readers to [18].

\section{Definition 2.10}

Let $\left(\boldsymbol{T} ; \odot, *, 1_{T}\right)$ be a GBE-semigroup. Then a subset $\emptyset \neq \boldsymbol{S}$ of $\boldsymbol{T}$ is called a left (resp. right) deductive system if the following conditions are satisfied:

(i) $\boldsymbol{T} \odot S \subseteq S$ (resp. $(S \odot T \subseteq S)$ ),

(ii) $\forall s \in \boldsymbol{S}$ and $\forall t \in \boldsymbol{T}$ such that $s * t \in \boldsymbol{S} \Longrightarrow t \in \boldsymbol{S}$.

Note that a deductive system is one which is both left as well as right deductive system.

Let us give an example in order to understand deductive systems and for this we refer the readers to the source [18].

\section{Example 2.11}

Let $N=\left\{1_{N}, x, y, z, t\right\}$ and define " $\odot$ " and “*” in $N$ in the following way:

\begin{tabular}{|c|c|c|c|c|c|}
\hline$\odot$ & $1_{N}$ & $x$ & $y$ & $z$ & $t$ \\
\hline $1_{N}$ & $1_{N}$ & $1_{N}$ & $1_{N}$ & $1_{N}$ & $1_{N}$ \\
\hline$x$ & $1_{N}$ & $1_{N}$ & $1_{N}$ & $1_{N}$ & $y$ \\
\hline$y$ & $1_{N}$ & $1_{N}$ & $1_{N}$ & $1_{N}$ & $1_{N}$ \\
\hline$z$ & $1_{N}$ & $1_{N}$ & $1_{N}$ & $1_{N}$ & $1_{N}$ \\
\hline$t$ & $1_{N}$ & $1_{N}$ & $1_{N}$ & $1_{N}$ & $1_{N}$ \\
\hline
\end{tabular}

\begin{tabular}{|c|c|c|c|c|c|}
\hline$*$ & $1_{N}$ & $x$ & $y$ & $z$ & $t$ \\
\hline $1_{N}$ & $1_{N}$ & $x$ & $y$ & $1_{N}$ & $t$ \\
\hline$x$ & $1_{N}$ & $1_{N}$ & $y$ & $1_{N}$ & $1_{N}$ \\
\hline$y$ & $1_{N}$ & $x$ & $1_{N}$ & $1_{N}$ & $t$ \\
\hline$z$ & $1_{N}$ & $x$ & $y$ & $1_{N}$ & $t$ \\
\hline$t$ & $1_{N}$ & $x$ & $y$ & $1_{N}$ & $1_{N}$ \\
\hline
\end{tabular}

Then it is easy to check that $\left(N ; \odot, *, 1_{N}\right)$ is a GBE-semigroup. Assume that $X=\left\{1_{N}, z\right\}$, then $\boldsymbol{X}$ is $\boldsymbol{L D S}$ and $\boldsymbol{R D S}$ as well of $\boldsymbol{N}$. Now assume that $\boldsymbol{Y}=\left\{1_{N}, t\right\}$, then $\boldsymbol{Y}$ is not an $\boldsymbol{L D S}$ of $\boldsymbol{N}$, as $x \odot t=y \notin \boldsymbol{Y}$ or $1_{N} * z=1_{N} \in \boldsymbol{Y}$ but $z \notin \boldsymbol{Y}$. 


\section{MAIN RESULTS THROUGH DEDUCTIVE SYSTEMS}

In this part, we define homomorphisms between two generalized BE-semigroups and investigate some relevant properties of homomorphisms and deductive systems. We show that every deductive system defines a congruence relation on a generalized BE-semigroup. Furthermore, we show that once we get the said congruence relation we shall obtain the factor generalized BE-semigroup. We start with the following definition.

\section{Definition 3.1}

Let $\left(\boldsymbol{R} ; \odot, *, 1_{\boldsymbol{R}}\right)$ and $\left(\boldsymbol{T} ; \odot,{ }^{*}, 1_{\boldsymbol{T}}\right)$ be two GBE-semigroups. Then a mapping $\varphi: \boldsymbol{R} \rightarrow \boldsymbol{T}$ is called a homomorphism if for all $r, s \in \boldsymbol{R}$,

$$
\varphi(r * s)=\varphi(r) * \varphi(s) \text { and } \varphi(r \odot s)=\varphi(r) \odot \varphi(s) .
$$

A homomorphism $\varphi$ is known as an epimorphism (resp. monomorphism) if it is onto (resp. one-one). A homomorphism which is both one-one and onto is called an isomorphism. Let $\varphi: \boldsymbol{R} \rightarrow \boldsymbol{T}$ be a homomorphism then kernel of $\varphi$ is denoted by $\boldsymbol{K e r}(\varphi)$ and is defined as:

$$
\operatorname{Ker}(\varphi)=\left\{u \in \boldsymbol{R}: \varphi(u)=1_{T}\right\}
$$

and the image of $\varphi$ is denoted by $\operatorname{Im}(\varphi)$ and is defined as:

$$
\operatorname{Im}(\varphi)=\{\varphi(u): u \in \boldsymbol{R}\} .
$$

The set of all homomorphisms from a GBE-semigroup $\boldsymbol{R}$ to a GBE-semigroup $\boldsymbol{T}$ is represented by the symbol $\operatorname{Hom}(\boldsymbol{R}, \boldsymbol{T})$.

Let us give some examples in order to understand the notion of homomorphisms.

\section{Example 3.2}

(i) The identity function on any GBE-semigroup is always an isomorphism.

(ii) Assume $\boldsymbol{L}=\left\{1_{L}, a, b, c\right\}$ and $\boldsymbol{M}=\left\{1_{M}, x, y, z, t\right\}$ be sets with the tables given below:

\begin{tabular}{|c|c|c|c|c|}
\hline$\odot$ & $1_{L}$ & $a$ & $b$ & $c$ \\
\hline $1_{\boldsymbol{L}}$ & $1_{\boldsymbol{L}}$ & $1_{\boldsymbol{L}}$ & $1_{\boldsymbol{L}}$ & $1_{\boldsymbol{L}}$ \\
\hline$a$ & $1_{\boldsymbol{L}}$ & $1_{\boldsymbol{L}}$ & $1_{\boldsymbol{L}}$ & $1_{\boldsymbol{L}}$ \\
\hline$b$ & $1_{\boldsymbol{L}}$ & $1_{\boldsymbol{L}}$ & $1_{\boldsymbol{L}}$ & $a$ \\
\hline$c$ & $1_{\boldsymbol{L}}$ & $1_{\boldsymbol{L}}$ & $1_{\boldsymbol{L}}$ & $1_{\boldsymbol{L}}$ \\
\hline
\end{tabular}

\begin{tabular}{|c|c|c|c|c|}
\hline$*$ & $1_{L}$ & $a$ & $b$ & $c$ \\
\hline $1_{L}$ & $1_{L}$ & $1_{L}$ & $1_{L}$ & $1_{L}$ \\
\hline$a$ & $1_{L}$ & $1_{L}$ & $1_{L}$ & $1_{L}$ \\
\hline$b$ & $1_{L}$ & $a$ & $1_{L}$ & $a$ \\
\hline$c$ & $1_{L}$ & $1_{L}$ & $1_{L}$ & $1_{L}$ \\
\hline
\end{tabular}




\begin{tabular}{|c|c|c|c|c|c|}
\hline$\odot$ & $1_{M}$ & $x$ & $y$ & $z$ & $t$ \\
\hline $1_{M}$ & $1_{M}$ & $1_{M}$ & $1_{M}$ & $1_{M}$ & $1_{M}$ \\
\hline$x$ & $1_{M}$ & $1_{M}$ & $1_{M}$ & $1_{M}$ & $1_{M}$ \\
\hline$y$ & $1_{M}$ & $1_{M}$ & $1_{M}$ & $1_{M}$ & $1_{M}$ \\
\hline$z$ & $1_{M}$ & $1_{M}$ & $1_{M}$ & $1_{M}$ & $1_{M}$ \\
\hline$t$ & $1_{M}$ & $1_{M}$ & $1_{M}$ & $1_{M}$ & $t$ \\
\hline
\end{tabular}

\begin{tabular}{|c|c|c|c|c|c|}
\hline$*$ & $1_{M}$ & $x$ & $y$ & $z$ & $t$ \\
\hline $1_{M}$ & $1_{M}$ & $y$ & $y$ & $1_{M}$ & $1_{M}$ \\
\hline$x$ & $1_{M}$ & $1_{M}$ & $1_{M}$ & $z$ & $1_{M}$ \\
\hline$y$ & $1_{M}$ & $1_{M}$ & $1_{M}$ & $1_{M}$ & $t$ \\
\hline$z$ & $1_{M}$ & $x$ & $y$ & $1_{M}$ & $1_{M}$ \\
\hline$t$ & $1_{M}$ & $1_{M}$ & $1_{M}$ & $1_{M}$ & $1_{M}$ \\
\hline
\end{tabular}

Then it is easy to verify that $\left(\boldsymbol{L} ; \odot, *, 1_{\boldsymbol{L}}\right)$ and $\left(\boldsymbol{M} ; \odot,{ }^{*}, 1_{\boldsymbol{M}}\right)$ are GBE-semigroups.

Let us define $\varphi: \boldsymbol{L} \rightarrow \boldsymbol{M}$ by the following rules:

$$
\varphi\left(1_{L}\right)=1_{M}, \varphi(a)=1_{M}, \varphi(b)=1_{M} \text { and } \varphi(c)=z .
$$

Then one can easily check that $\varphi$ is a homomorphism from $\left(\boldsymbol{L} ; \odot, *, 1_{\boldsymbol{L}}\right)$ into $\left(\boldsymbol{M} ; \odot,{ }^{*}, 1_{\boldsymbol{M}}\right)$.

Let us state and prove some properties. These properties are true in case of BE-semigroups and we convert them into GBE-semigroups.

\section{Theorem 3.3}

Suppose that $\left(\boldsymbol{W} ; \odot_{1}, *_{1}, 1_{\boldsymbol{W}}\right)$ and $\left(\boldsymbol{M} ; \odot_{2}, *_{2}, 1_{\boldsymbol{M}}\right)$ are GBE-semigroups. If $\underline{\boldsymbol{R}}$ is a left (resp. right) deductive system of $\boldsymbol{M}$ and if $\varphi \in \operatorname{Hom}(\boldsymbol{W}, \boldsymbol{M})$ then $\varphi^{-1}(\underline{R})$ is a left (resp. right) deductive system of $\boldsymbol{W}$ and $\operatorname{Ker}(\varphi) \subseteq \varphi^{-1}(\underline{R})$.

\section{Proof.}

Let us assume that $\underline{\boldsymbol{R}}$ is a left deductive system of $\boldsymbol{M}$. Let $v \in \boldsymbol{W}$ and $\hat{e} \in \varphi^{-1}(\underline{\boldsymbol{R}})$, then $\varphi(\hat{e}) \in \underline{R}$. Now $\varphi\left(v \odot_{1} \hat{e}\right)=\varphi(v) \bigodot_{2} \varphi(\hat{e})$ so it follows that $\varphi\left(v \odot_{1} \hat{e}\right) \in \underline{R}$ because $\underline{R}$ is a left deductive system. In other words, $v \odot_{1} \hat{e} \in \varphi^{-1}(\underline{\boldsymbol{R}})$. Hence $\boldsymbol{W} \odot_{1} \varphi^{-1}(\underline{\boldsymbol{R}}) \subseteq \varphi^{-1}(\underline{\boldsymbol{R}})$. Furthermore, let us suppose that $v, \hat{e} \in \boldsymbol{W}$ are such that $\hat{e} \in \varphi^{-1}(\underline{R})$ and $\hat{e} *_{1} v \in \varphi^{-1}(\underline{R})$, then $\varphi(\hat{e}) \in \underline{\boldsymbol{R}}$ and $\varphi\left(\hat{e} *_{1} v\right)=\varphi(\hat{e}) *_{2} \varphi(v) \in \underline{\boldsymbol{R}}$. As by assumption $\underline{\boldsymbol{R}}$ is a left deductive system so we get $\varphi(v) \in \underline{\boldsymbol{R}}$, i.e. $v \in \varphi^{-1}(\underline{\boldsymbol{R}})$. Hence $\varphi^{-1}(\underline{\boldsymbol{R}})$ is a left deductive system of $\boldsymbol{W}$. As $\left\{1_{\boldsymbol{M}}\right\} \subseteq \underline{\boldsymbol{R}}$ so it follows that $\varphi^{-1}\left(\left\{1_{M}\right\}\right) \subseteq \varphi^{-1}(\underline{R})$. It implies that $\operatorname{Ker}(\varphi) \subseteq \varphi^{-1}(\underline{\boldsymbol{R}})$. In the same way, we may prove the required result for the right deductive system. 


\section{Theorem 3.4}

Let $\left(\boldsymbol{L} ; \odot_{1}, *_{1}, 1_{\boldsymbol{L}}\right)$ and $\left(\boldsymbol{M} ; \odot_{2}, *_{2}, 1_{\boldsymbol{M}}\right)$ be GBE-semigroups. Suppose that $\varphi: \boldsymbol{L} \rightarrow \boldsymbol{M}$ is an epimorphism and assume that $\underline{\boldsymbol{R}}$ is a left (resp. right) deductive system of $\boldsymbol{L}$. Then $\varphi(\underline{\boldsymbol{R}})$ is a left (resp. right) deductive system of $\boldsymbol{M}$.

\section{Proof.}

Let $\tilde{n} \in \varphi(\underline{\boldsymbol{R}})$ and $s \in \boldsymbol{M}$. Since $\varphi$ is surjective, there exists $w \in \boldsymbol{L}$ such that $\varphi(w)=s$ and $\varphi(v)=\tilde{n}$ for some $v \in \underline{\boldsymbol{R}}$, by definition of $\varphi(\underline{\boldsymbol{R}})$. Thus $w \odot_{1} v \in \underline{\boldsymbol{R}}$ implies that $\varphi\left(w \odot_{1} v\right) \in$ $\varphi(\underline{\boldsymbol{R}}) \Longrightarrow \varphi(w) \odot_{2} \varphi(v) \in \varphi(\underline{\boldsymbol{R}}) \Rightarrow s \odot_{2} \tilde{n} \in \varphi(\underline{\boldsymbol{R}})$. Therefore, $\boldsymbol{M} \odot_{2} \varphi(\underline{\boldsymbol{R}}) \subseteq \varphi(\underline{\boldsymbol{R}})$. Furthermore, let $w \in \varphi(\underline{R})$ and $s \in \boldsymbol{M}$ be such that $w *_{2} s \in \varphi(\underline{\boldsymbol{R}})$. As $\psi$ is surjective so there exists $\tilde{n} \in \boldsymbol{L}$ such that $\varphi(\tilde{n})=s$ and $\varphi(v)=w$ for some $v \in \underline{\boldsymbol{R}}$, by definition of $\varphi(\underline{\boldsymbol{R}})$. As $w *_{2} s \in \varphi(\underline{\boldsymbol{R}}) \Longrightarrow \varphi(v) *_{2} \varphi(\tilde{n}) \in \varphi(\underline{\boldsymbol{R}}) \Longrightarrow \varphi\left(v *_{1} \tilde{n}\right) \in \varphi(\underline{\boldsymbol{R}})$.Therefore, by definition of $\varphi(\underline{\boldsymbol{R}})$, it implies that $v *_{1} \tilde{n} \in \underline{\boldsymbol{R}}$. As $\underline{\boldsymbol{R}}$ is a deductive system, so it follows that $\tilde{n} \in \underline{\boldsymbol{R}}$. Therefore, $s=\varphi(\tilde{n}) \in \varphi(\underline{\boldsymbol{R}})$. Hence, $\varphi(\underline{\boldsymbol{R}})$ is a left deductive system of $\boldsymbol{M}$. In the same way, we may have the desired case for the right deductive system.

Note that a GBE-semigroup $\left(\boldsymbol{K} ; \odot, *, 1_{\boldsymbol{K}}\right)$ is considered to be a self-distributive GBEsemigroup when $\boldsymbol{K}$ is self-distributive GBE-algebra.

Let us define left (resp. right) congruence relations in a GBE-semigroup.

\section{Definition 3.5}

Assume that $\left(\boldsymbol{M} ; \odot, *, 1_{\boldsymbol{M}}\right)$ is a GBE-semigroup. A relation $\rho$ on $\boldsymbol{M}$ is called left compatible if $\forall l, m, n \in \boldsymbol{M}$ such that $(l, m) \in \rho$ implies that $(n \odot l, n \odot m) \in \rho$ and $(n * l, n * m) \in \rho$. Similarly a relation $\rho$ on $\boldsymbol{M}$ is called right compatible if $\forall l, m, n \in \boldsymbol{M}$ such $(l, m) \in \rho$ implies that $(l \odot n, m \odot n) \in \rho$ and $(l * n, m * n) \in \rho$.

\section{Definition 3.6}

A relation $\rho$ on a GBE-semigroup $\left(S ; \odot, *, 1_{S}\right)$ is known as compatible if $\forall x, y, z, t \in S$ such that $(x, y),(z, t) \in \rho$ implies that $(x \odot z, y \odot t),(x * z, y * t) \in \rho$.

Note that if a relation is left compatible as well as equivalence relation, then it is called a left congruence relation. In the same way if a relation is right compatible as well as equivalence relation, then it is called a right congruence relation. Moreover, if a relation is compatible as well as equivalence relation, then it is called a congruence relation.

We now give some examples in order to understand the above notions.

\section{Example 3.7}

(i) Let $\left(\boldsymbol{T} ; \odot, *, 1_{T}\right)$ be a GBE-semigroup then it is easy to see that $\nabla=\boldsymbol{T} \times \boldsymbol{T}$ and $\Delta=\{(r, r): r \in \boldsymbol{T}\}$ are congruence relations on $\boldsymbol{T}$.

(ii) Assume that $\boldsymbol{K}=\left\{1_{K}, u, v, w\right\}$ and the operations " $*$ " and " $\odot$ " are defined below: 


\begin{tabular}{|c|c|c|c|c|}
\hline$\odot$ & $1_{\boldsymbol{K}}$ & $u$ & $v$ & $w$ \\
\hline $1_{\boldsymbol{K}}$ & $1_{\boldsymbol{K}}$ & $1_{\boldsymbol{K}}$ & $1_{\boldsymbol{K}}$ & $1_{\boldsymbol{K}}$ \\
\hline$u$ & $1_{\boldsymbol{K}}$ & $1_{\boldsymbol{K}}$ & $1_{\boldsymbol{K}}$ & $1_{\boldsymbol{K}}$ \\
\hline$v$ & $1_{\boldsymbol{K}}$ & $1_{\boldsymbol{K}}$ & $1_{\boldsymbol{K}}$ & $u$ \\
\hline$w$ & $1_{\boldsymbol{K}}$ & $1_{\boldsymbol{K}}$ & $1_{\boldsymbol{K}}$ & $1_{\boldsymbol{K}}$ \\
\hline
\end{tabular}

\begin{tabular}{|c|c|c|c|c|}
\hline$*$ & $1_{\boldsymbol{K}}$ & $u$ & $v$ & $w$ \\
\hline $1_{\boldsymbol{K}}$ & $1_{\boldsymbol{K}}$ & $1_{\boldsymbol{K}}$ & $1_{\boldsymbol{K}}$ & $1_{\boldsymbol{K}}$ \\
\hline$u$ & $1_{\boldsymbol{K}}$ & $1_{\boldsymbol{K}}$ & $1_{\boldsymbol{K}}$ & $1_{\boldsymbol{K}}$ \\
\hline$v$ & $1_{\boldsymbol{K}}$ & $u$ & $1_{\boldsymbol{K}}$ & $u$ \\
\hline$w$ & $1_{\boldsymbol{K}}$ & $1_{\boldsymbol{K}}$ & $1_{\boldsymbol{K}}$ & $1_{\boldsymbol{K}}$ \\
\hline
\end{tabular}

Then one can easily see that $\left(\boldsymbol{K} ; \odot, *, 1_{\boldsymbol{K}}\right)$ is a GBE-semigroup. Now let us assume that $\theta=\Delta \cup\left\{\left(u, 1_{K}\right),\left(1_{K}, u\right)\right\}$ then it is easy to check that $\theta$ is a congruence relation on $\boldsymbol{K}$.

We now state and prove some properties. The below result provides us equivalent conditions for congruence relations in GBE-semigroups. The result is true in case of semigroups and we convert it into GBE-semigroups.

\section{Proposition 3.8}

Assume that $\rho$ is an equivalence relation on a GBE-semigroup $\left(Z ; \odot, *, 1_{Z}\right)$. Then $\rho$ is a congruence relation if and only if it is both left and right congruence relation on the GBEsemigroup $\boldsymbol{Z}$.

\section{Proof.}

Let us assume that $\rho$ is a congruence relation on $\boldsymbol{Z}$. In order to show that $\rho$ is both left as well as right congruence relation, let $x, y, z \in Z$ be such that $(x, y) \in \rho$. Now $(z, z) \in \rho$, as $\rho$ is reflexive. Since $\rho$ is compatible, so we have $(z \odot x, z \odot y),(z * x, z * y) \in \rho$. Thus it follows that $\rho$ is a left congruence relation. In the same way, we can show that $(x \odot z, y \odot z)$, $(x * z, y * z) \in \rho$. Thus, it follows that $\rho$ is a right congruence relation.

Let us suppose that $\rho$ is both left and right congruence relation on $\boldsymbol{Z}$. In order to show that $\rho$ is a congruence relation, let $x, y, z, t \in \boldsymbol{Z}$ be such that $(x, y),(z, t) \in \rho$. Now $(x \odot z, y \odot z)$, $(x * z, y * z) \in \rho$, as $\rho$ is right compatible and $(y \odot z, y \odot t),(y * z, y * t) \in \rho$, as $\rho$ is left compatible. By transitivity, we have $(x \odot z, y \odot t),(x * z, y * t) \in \rho$. This completes the proof.

We are now going to define a special type of relation in self-distributive GBE-semigroups through a deductive system.

\section{Definition 3.9}

Suppose $\boldsymbol{W}$ is a deductive system of a self-distributive GBE-semigroup $\left(\boldsymbol{L} ; \odot, *, 1_{\boldsymbol{L}}\right)$. Let $u, v \in \boldsymbol{L}$. A relation " $\sim \boldsymbol{W}$ " on $\boldsymbol{L}$ can be defined as under:

$$
u \sim \boldsymbol{W} v \Leftrightarrow u * v \in \boldsymbol{W} \text { and } v * u \in \in \boldsymbol{W} .
$$

The following result shows that the above defined relation is a congruence relation. The result is true in case of BE-semigroups and we convert it into GBE-semigroups. 


\section{Theorem 3.10}

Suppose $\boldsymbol{W}$ is a deductive system of a self-distributive $\operatorname{GBE}$-semigroup $\left(\boldsymbol{L} ; \odot, *, 1_{\boldsymbol{L}}\right)$. Then $\sim W$ is a equivalence relation on $\boldsymbol{L}$.

\section{Proof.}

Let $d \in \boldsymbol{W}$. As $\boldsymbol{W}$ is a deductive system of $\boldsymbol{L}$ and furthermore $1_{\boldsymbol{L}} \in \boldsymbol{L}$, then by axiom (i) of deductive systems we have, $\boldsymbol{L} \odot \boldsymbol{W} \subseteq \boldsymbol{W} \Rightarrow 1_{\boldsymbol{L}} \odot d \in \boldsymbol{W} \Rightarrow 1_{\boldsymbol{L}} \in \boldsymbol{W}$. Suppose $s \in \boldsymbol{L}$, then $s * s$ $=1_{L} \in W$ and therefore, $s \sim W S \Rightarrow \sim W$ is reflexive. Now if $s \sim W \tilde{e}$, for any $s, \tilde{e} \in \boldsymbol{L}$, then it is obvious from the definition that $\tilde{e} \sim W s$. This means that $\sim W$ is symmetric. Let $s, \tilde{e}, v \in \boldsymbol{L} \exists$ $s \sim \boldsymbol{W} \tilde{e}$ and $\tilde{e} \sim \boldsymbol{W} v$, then $s * \tilde{e} \in \boldsymbol{W}, \tilde{e} * s \in \boldsymbol{W}$ and $\tilde{e} * v \in \boldsymbol{W}, v * \tilde{e} \in \boldsymbol{W}$.

Now,

$$
\begin{aligned}
(\tilde{e} * v) *((s * \tilde{e}) *(s * v)) & =(\tilde{e} * v) *(s *(\tilde{e} * v)) & & (\because \text { by self-distributive law }) \\
& =S *((\tilde{e} * v) *(\tilde{e} * v)) & & (\because \text { by (iv) of GBE-algebra }) \\
& =S * 1_{L} & & \left(\because s * S=1_{L}\right) \\
& =1_{L} \in \boldsymbol{W} & & \left(\because S * 1_{L}=1_{L}\right)
\end{aligned}
$$

$\Longrightarrow(s * \tilde{e}) *(s * v) \in \boldsymbol{W}$ (since by condition (ii) of deductive systems) and so $s * v \in \boldsymbol{W}$. Similarly,

$$
\begin{aligned}
(\tilde{e} * s) *((v * \tilde{e}) *(v * s)) & =(\tilde{e} * s) *(v *(\tilde{e} * s)) & & (\because \text { By self-distributive law }) \\
& =v *((\tilde{e} * s) *(\tilde{e} * s)) & & (\because \text { by axiom (iv) of GBE-algebra }) \\
& =v * 1_{L} & & \left(\because s * s=1_{L}\right) \\
& =1_{L} \in \boldsymbol{W} & & \left(\because s * 1_{L}=1_{L}\right)
\end{aligned}
$$

This implies that $(r * \tilde{e}) *(v * s) \in \boldsymbol{W}$ (since by (ii) of deductive systems) and so $r * s \in$ $\boldsymbol{W}$. Thus, $s \sim \boldsymbol{W} v$. This follows that $\sim \boldsymbol{W}$ is transitive. This proves what we wanted.

Let us suppose that $\left(\boldsymbol{L} ; \odot, *, 1_{\boldsymbol{L}}\right)$ is a self-distributive GBE-semigroup and $\boldsymbol{W}$ a deductive system of $\boldsymbol{L}$. Let us consider the above equivalence relation on $\boldsymbol{L}$, then the equivalence class corresponding to $s \in \boldsymbol{L}$ is denoted by $\boldsymbol{W}^{s}$. Furthermore, the set of all equivalence classes in $\boldsymbol{L}$ is represented by $\boldsymbol{L} / \boldsymbol{W}$. Symbolically,

$$
\boldsymbol{W}^{s}=\left\{r \in \boldsymbol{L}, v \sim \boldsymbol{W}_{\varepsilon}\right\} \text { and } \boldsymbol{L} / \boldsymbol{W}=\left\{\boldsymbol{W}^{s}: s \in \boldsymbol{L}\right\} .
$$

We can define two binary operations "○” and "®" in $\boldsymbol{L} / \boldsymbol{W}$ as follows:

$$
\boldsymbol{W}^{w} \odot \boldsymbol{W}^{\tilde{n}}=\boldsymbol{W}^{w} \odot \tilde{n} \text { and } \boldsymbol{W}^{w} \circledast \boldsymbol{W}^{\tilde{n}}=\boldsymbol{W}^{w} * \tilde{n} \forall \boldsymbol{W}^{w}, \boldsymbol{W}^{\tilde{n}} \in \boldsymbol{L} / \boldsymbol{W} \text {. }
$$


Now in order to show $\sim W$ is a congruence relation on $L$, we prove that $\sim W$ is compatible. For this assume $\hat{e}, \dot{n}, \bar{g}, \dot{w} \in \boldsymbol{L} \ni \hat{e} \sim W \dot{n}$ and $\bar{g} \sim W \dot{w}$ then $\hat{e} * \dot{n} \in \boldsymbol{W}, \dot{n} * \hat{e} \in \boldsymbol{W}$ and $\bar{g} * \dot{w} \in \boldsymbol{W}$, $\dot{w} * \bar{g} \in W$. We have to prove that $\hat{e} * \bar{g} \sim W \dot{n} * \dot{w}$ and $\hat{e} \odot \bar{g} \sim W \dot{n} \odot \dot{w}$. Here

$\hat{e} \sim \boldsymbol{W} \dot{n}$ and $\bar{g} \sim \boldsymbol{W} \dot{w} \Rightarrow \boldsymbol{W}^{\hat{e}}=\boldsymbol{W}^{\dot{n}}$ and $\boldsymbol{W}^{\bar{g}}=\boldsymbol{W}^{\dot{w}} \Rightarrow \boldsymbol{W}^{\hat{e}} \circledast \boldsymbol{W}^{\bar{g}}=\boldsymbol{W}^{\dot{n}} \circledast \boldsymbol{W}^{\dot{w}}$ and $\boldsymbol{W}^{\hat{e}} \odot \boldsymbol{W}^{\bar{g}}=\boldsymbol{W}^{\tilde{n}} \odot \boldsymbol{W}^{\dot{w}} \Rightarrow \boldsymbol{W}^{\hat{e}} * \bar{g}=\boldsymbol{W}^{\dot{n}} *{ }^{\dot{w}}$ and $\boldsymbol{W}^{\hat{e}} \odot \bar{g}=\boldsymbol{W}^{\dot{n}} \odot{ }^{\dot{w}} \Rightarrow \hat{e} * \bar{g} \sim \boldsymbol{W} \dot{n} * \dot{w}$ and $\hat{e} \odot \bar{g}_{\sim W} \dot{n} \odot \dot{w}$.

This shows that $\sim W$ is a congruence relation.

Let us prove some relevant results. These results are true in case of BE-semigroups and we convert them into GBE-semigroups.

\section{Theorem 3.11}

Suppose $\left(\boldsymbol{L} ; \odot, *, 1_{\boldsymbol{L}}\right)$ is a GBE-semigroup and moreover it is self-distributive. If $\boldsymbol{W}$ is a deductive system of $\boldsymbol{L}$, then $\boldsymbol{L} / \boldsymbol{W}$ is a self-distributive GBE-semigroup under the above binary operations, i.e.

$$
\boldsymbol{W}^{w} \odot \boldsymbol{W}^{\tilde{n}}=\boldsymbol{W}^{w} \odot \tilde{n} \text { and } \boldsymbol{W}^{w} \odot \boldsymbol{W}^{\tilde{n}}=\boldsymbol{W}^{w} * \tilde{n} \forall \boldsymbol{W}^{w}, \boldsymbol{W}^{\tilde{n}} \in \boldsymbol{L} / \boldsymbol{W} .
$$

\section{Proof.}

\section{Well defined:}

Let $\boldsymbol{W}^{w}=\boldsymbol{W}^{\tilde{n}}$ and $\boldsymbol{W}^{\dot{u}}=\boldsymbol{W}^{\hat{e}}$ then $w * \tilde{n} \in \boldsymbol{W}, \tilde{n} * w \in \boldsymbol{W}$ and $\dot{u} * \hat{e} \in \boldsymbol{W}, \hat{e} * \dot{u} \in \boldsymbol{W}$. Now

$$
(w \odot u) *(w \odot \hat{e})=w \odot(\dot{u} * \hat{e}) \quad(\because \odot \text { is distributive over } *)
$$

and

$$
w \odot(\dot{u} * \hat{e}) \in \boldsymbol{W}
$$

$(\because \boldsymbol{W}$ is left deductive system)

Moreover,

and

$$
(w \odot \hat{e}) *(w \odot u)=w \odot(\hat{e} * u) \quad(\because \odot \text { is distributive over } *)
$$

$$
w \odot(\hat{e} * u) \in \boldsymbol{W} \quad(\because \boldsymbol{W} \text { is left deductive system })
$$

Therefore, $w \odot \dot{u} \sim w w \odot \hat{e}$. Furthermore,

and

$$
(w \odot \hat{e}) *(\tilde{n} \odot \hat{e})=(w * \tilde{n}) \odot \hat{e} \quad(\because \odot \text { is distributive over } *)
$$

Moreover,

$$
(w * \tilde{n}) \odot \hat{e} \in \boldsymbol{W}
$$

( $\because \boldsymbol{W}$ is right deductive system)

and

$$
(\tilde{n} \odot \hat{e}) *(w \odot \hat{e})=(\tilde{n} * w) \odot \hat{e}
$$$$
(\because \odot \text { is distributive over } *)
$$

$$
(\tilde{n} * w) \odot \hat{e} \in \boldsymbol{W}
$$

( $\because \boldsymbol{W}$ is right deductive system)

Thus, $w \odot \hat{e}_{\sim} w \tilde{n} \odot \hat{e}$. 


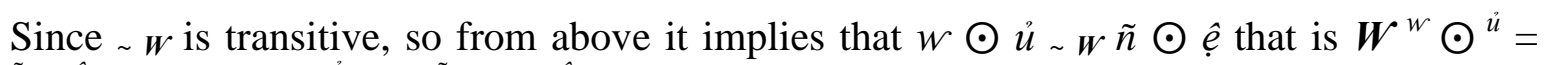
$\boldsymbol{W}^{\tilde{n}} \odot \stackrel{\hat{e}}{\Rightarrow} \boldsymbol{W}^{w} \odot \boldsymbol{W}^{\dot{u}}=\boldsymbol{W}^{\tilde{n}} \odot \boldsymbol{W}^{\hat{e}}$. This shows that "○" is well-defined.

Now,

$$
\begin{aligned}
& (\hat{u} * \hat{e}) *((w * \hat{u}) *(w * \hat{e}))=(\dot{u} * \hat{e}) *((w *(\hat{u} * \hat{e})) \quad(\because \boldsymbol{L} \text { is a self-distributive }) \\
& =w *((\dot{u} * \hat{e}) *(\dot{u} * \hat{e})) \quad(\because \text { by exchange law }) \\
& =W * 1_{L} \\
& =1_{L}
\end{aligned}
$$

and $1_{\boldsymbol{L}} \in \boldsymbol{W}$ so that $(\hat{u} * \hat{e}) *((w * \hat{u}) *(w * \hat{e})) \in \boldsymbol{W}$. As $\boldsymbol{W}$ is a deductive system, so it follows that $(w * u) *(w * \hat{e}) \in \boldsymbol{W}$.

Now,

$$
\begin{aligned}
& (\hat{e} * \hat{u}) *((w * \hat{e}) *(w * \hat{u}))=(\hat{e} * \mathfrak{u}) *((w *(\hat{e} * \hat{u})) \quad(\because \boldsymbol{L} \text { is a self-distributive }) \\
& =w *((\hat{e} * u) *(\hat{e} * u ̉)) \quad(\because \text { by exchange law }) \\
& =W * 1_{L} \\
& =1_{L}
\end{aligned}
$$

and $1_{\boldsymbol{L}} \in \boldsymbol{W}$ so that $(\hat{e} * \hat{u}) *((w * \hat{e}) *(w * \hat{u})) \in \boldsymbol{W}$. As $\boldsymbol{W}$ is a deductive system, so it follows that $(w * \hat{e}) *(w * u) \in W$. Therefore, $w * \dot{u} \sim W w * \hat{e}$.

Further,

$$
\begin{array}{rlrl}
(\tilde{n} * w) *((w * \hat{e}) *(\tilde{n} * \hat{e})) & =(w * \hat{e}) *((\tilde{n} * w) *(\tilde{n} * \hat{e})) & (\because \text { by exchange law }) \\
& =(w * \hat{e}) *((\tilde{n} *(w * \hat{e})) & (\because L \text { is a self-distributive }) \\
& =\tilde{n} *((w * \hat{e}) *(w * \hat{e})) & & (\because \text { by exchange law }) \\
& =\tilde{n} * 1_{L} \\
& =1_{L}
\end{array}
$$

and $1_{\boldsymbol{L}} \in \boldsymbol{W}$ so that $(\tilde{n} * w) *((w * \hat{e}) *(\tilde{n} * \hat{e})) \in \boldsymbol{W}$. As $\boldsymbol{W}$ is a deductive system, so it follows that $(w * \hat{e}) *(\tilde{n} * \hat{e})) \in \boldsymbol{W}$.

Furthermore,

$$
\begin{aligned}
(w * \tilde{n}) *((\tilde{n} * \hat{e}) *(w * \hat{e})) & =(\tilde{n} * \hat{e}) *((w * \tilde{n}) *(w * \hat{e})) & & (\because \text { by exchange law }) \\
& =(\tilde{n} * \hat{e}) *((w *(\tilde{n} * \hat{e})) & & (\because \boldsymbol{L} \text { is a self-distributive }) \\
& =w *((\tilde{n} * \hat{e}) *(\tilde{n} * \hat{e})) & & (\because \text { by exchange law })
\end{aligned}
$$




$$
\begin{aligned}
& =w * 1_{L} \\
& =1_{L}
\end{aligned}
$$

and $1_{L} \in \boldsymbol{W}$ so that $(W * \tilde{n}) *((\tilde{n} * \hat{e}) *(w * \hat{e})) \in \boldsymbol{W}$. As $\boldsymbol{W}$ is a deductive system, so it follows that $(\tilde{n} * \hat{e}) *(W * \hat{e}) \in W$. Thus, $w * \hat{e} \sim W \tilde{n} * \hat{e}$. As $\sim W$ is transitive, so from the above it follows that $w * \dot{u} \sim \boldsymbol{W}_{\tilde{n}} * \hat{e}$. Thus, $\boldsymbol{W}^{\dot{w}} *{ }^{\dot{u}}=\boldsymbol{W}^{\dot{\tilde{n}}} * \stackrel{\hat{e}}{\Rightarrow} \boldsymbol{W}^{w} \circledast \boldsymbol{W}^{\dot{u}}=\boldsymbol{W}^{\tilde{n}} \circledast \boldsymbol{W}^{\hat{e}}$. This implies that " $\circledast$ " is well-defined.

We now prove $\left(\boldsymbol{L} / \boldsymbol{W} ; \circledast, \boldsymbol{W}^{1} \boldsymbol{L}\right)$ is a GBE-algebra. Here

(i) $\boldsymbol{W}^{\dot{w}} \circledast \boldsymbol{W}^{\dot{w}}=\boldsymbol{W}^{\dot{w}} *{ }^{\dot{w}}=\boldsymbol{W}^{1} \boldsymbol{L} \forall \boldsymbol{W}^{\dot{w}} \in \boldsymbol{L} / \boldsymbol{W}$.

(ii) $\boldsymbol{W}^{\dot{w}} \circledast \boldsymbol{W}^{1}{ }_{\boldsymbol{L}}=\boldsymbol{W}^{\dot{w}} *{ }_{\boldsymbol{L}}=\boldsymbol{W}^{1}{ }_{\boldsymbol{L}} \forall \boldsymbol{W}^{\dot{w}} \in \boldsymbol{L} / \boldsymbol{W}$.

(iii) $\boldsymbol{W}^{\dot{w}} \circledast\left(\boldsymbol{W}^{t} \circledast \boldsymbol{W}^{v}\right)=\boldsymbol{W}^{\dot{w}} \circledast\left(\boldsymbol{W}^{t} *^{v}\right)=\boldsymbol{W}^{\dot{w}} *{ }^{(t} * *^{v)}=\boldsymbol{W}^{t} *{ }^{(\dot{w}} *{ }^{v)}=\boldsymbol{W}^{t} \circledast \boldsymbol{W}^{\dot{w}} *^{r}=$ $\boldsymbol{W}^{t} \circledast\left(\boldsymbol{W}^{w} \circledast \boldsymbol{W}^{v}\right) \forall \boldsymbol{W}^{w}, \boldsymbol{W}^{t}, \boldsymbol{W}^{r} \in \boldsymbol{L} / \boldsymbol{W}$.

Thus $\left(\boldsymbol{L} / \boldsymbol{W} ; \circledast, \boldsymbol{W}^{1}{ }_{\boldsymbol{L}}\right)$ is a GBE-algebra. We now prove that $(\boldsymbol{L} / \boldsymbol{W} ; \bigcirc)$ is a semigroup.

\section{Closure Property:}

It is clear from the definition.

\section{Associative Law:}

Let $\boldsymbol{W}^{\dot{w}}, \boldsymbol{W}^{t}, \boldsymbol{W}^{v} \in \boldsymbol{L} / \boldsymbol{W}$, then

$\left(\boldsymbol{W}^{\dot{w}} \odot \boldsymbol{W}^{t}\right) \odot \boldsymbol{W}^{v}=\left(\boldsymbol{W}^{\dot{w}} \odot{ }^{t}\right) \odot \boldsymbol{W}^{v}=\boldsymbol{W}^{(\dot{w}} \odot^{t)} \odot{ }^{r}=\boldsymbol{W}^{\dot{w}} \odot^{(t} \odot{ }^{v)}=\boldsymbol{W}^{\dot{w}} \odot \boldsymbol{W}^{t} \odot^{r}=$ $\boldsymbol{W}^{w} \odot\left(\boldsymbol{W}^{t} \odot \boldsymbol{W}^{v}\right)$.

Hence, $(\boldsymbol{L} / \boldsymbol{W} ;$ ○) is a semigroup.

Now for any $\boldsymbol{W}^{w}, \boldsymbol{W}^{t}, \boldsymbol{W}^{v} \in \boldsymbol{L} / \boldsymbol{W}$, we obtain

$$
\begin{aligned}
& \boldsymbol{W}^{w} \odot\left(\boldsymbol{W}^{t} \odot \boldsymbol{W}^{v}\right)=\boldsymbol{W}^{w} \odot \boldsymbol{W}^{t} *^{r}=\boldsymbol{W}^{w} \odot^{(t} *^{v)}=\boldsymbol{W}^{(w} \odot^{t)} *^{(w} \odot^{r)}= \\
& \boldsymbol{W}^{(w} \odot{ }^{t)} \odot \boldsymbol{W}^{(w} \odot{ }^{r)}=\left(\boldsymbol{W}^{w} \odot \boldsymbol{W}^{t}\right) \odot\left(\boldsymbol{W}^{w} \odot \boldsymbol{W}^{v}\right) .
\end{aligned}
$$

Similarly,

$$
\begin{aligned}
& \left(\boldsymbol{W}^{w} \odot \boldsymbol{W}^{t}\right) \odot \boldsymbol{W}^{v}=\boldsymbol{W}^{w} *^{t} \odot \boldsymbol{W}^{v}=\boldsymbol{W}^{(w}{ }^{t}{ }^{t)} \odot{ }^{v}=\boldsymbol{W}^{(w} \odot{ }^{v)} *{ }^{(t} \odot^{v)}= \\
& \boldsymbol{W}^{(w} \odot{ }^{v)} \odot \boldsymbol{W}^{(t} \odot^{v)}=\left(\boldsymbol{W}^{w} \odot \boldsymbol{W}^{v}\right) \odot\left(\boldsymbol{W}^{t} \odot \boldsymbol{W}^{v}\right) .
\end{aligned}
$$

It implies that "○” is distributive over "®”. Thus $\boldsymbol{L} / \boldsymbol{W}$ is a GBE-semigroup. Now for selfdistributivity, suppose $\boldsymbol{W}^{w}, \boldsymbol{W}^{v}, \boldsymbol{W}^{t} \in \boldsymbol{L} / \boldsymbol{W}$, we obtain

$$
\left.\boldsymbol{W}^{w} \circledast\left(\boldsymbol{W}^{v} \circledast \boldsymbol{W}^{t}\right)=\boldsymbol{W}^{w} \circledast \boldsymbol{W}^{v} *^{t}=\boldsymbol{W}^{w} *^{(v} *^{t)}=\boldsymbol{W}^{(w} *^{v)} *^{(w} *^{t)}=\boldsymbol{W}^{(w} *^{r)} \circledast \boldsymbol{W}^{(w} *^{t}\right)
$$




$$
=\left(\boldsymbol{W}^{w} \circledast \boldsymbol{W}^{v}\right) \circledast\left(\boldsymbol{W}^{w} \circledast \boldsymbol{W}^{t}\right) .
$$

Thus, $\left(\boldsymbol{L} / \boldsymbol{W} ; \odot, \circledast, \boldsymbol{W}^{1} \boldsymbol{L}\right)$ is a self-distributive GBE-semigroup.

Note that the GBE-semigroup discussed in Theorem 3.11 is called quotient GBEsemigroup or factor GBE-semigroup. Moreover, we have

\section{Proposition 3.12}

Suppose $\left(\boldsymbol{G} ; \odot, *, 1_{\boldsymbol{G}}\right)$ is a self-distributive GBE-semigroup and moreover $\underline{\boldsymbol{R}}$ and $\boldsymbol{W}$ are deductive systems of $\boldsymbol{G}$. Further, suppose that $\underline{\boldsymbol{R}} \subset \boldsymbol{W}$, then

(i) $\underline{R}$ is also a deductive system of $\boldsymbol{W}$.

(ii) $\boldsymbol{W} / \underline{R}$ is a deductive system of $\boldsymbol{G} / \underline{R}$.

\section{Proof.}

(i) We need to show that $\boldsymbol{W} \odot \underline{\boldsymbol{R}} \subseteq \underline{\boldsymbol{R}}$ and $\underline{\boldsymbol{R}} \odot \boldsymbol{W} \subseteq \underline{\boldsymbol{R}}$ and $\forall \underline{u} \in \underline{\boldsymbol{R}}, \forall \dot{n} \in \boldsymbol{W} \ni u * \dot{n} \in \underline{\boldsymbol{R}}$ implies that $\dot{n} \in \underline{\boldsymbol{R}}$. Let $w \odot s \in \boldsymbol{W} \odot \underline{\boldsymbol{R}} \Rightarrow w \in \boldsymbol{W}$ and $s \in \underline{\boldsymbol{R}} \Rightarrow w \in \boldsymbol{G}$ and $s \in \underline{\boldsymbol{R}} \Rightarrow w \odot s$ $\in \boldsymbol{G} \odot \underline{\boldsymbol{R}} \subseteq \underline{\boldsymbol{R}} \Rightarrow w \odot s \in \underline{\boldsymbol{R}} \Rightarrow \boldsymbol{W} \odot \underline{\boldsymbol{R}} \subseteq \underline{\boldsymbol{R}}$. Now let $s \odot w \in \underline{\boldsymbol{R}} \odot \boldsymbol{W} \Rightarrow s \in \underline{\boldsymbol{R}}$ and $w \in \boldsymbol{W}$ implies that $s \in \underline{\boldsymbol{R}}$ and $w \in \boldsymbol{G} \Rightarrow s \odot w \in \underline{\boldsymbol{R}} \odot \boldsymbol{G} \subseteq \underline{\boldsymbol{R}} \Longrightarrow s \odot w \in \underline{\boldsymbol{R}} \Longrightarrow \underline{\boldsymbol{R}} \odot \boldsymbol{W} \subseteq \underline{\boldsymbol{R}}$.

Now for the second axiom of deductive systems, let $u \in \underline{R}$ and $\dot{n} \in \boldsymbol{W}$ be such that $u * \dot{n} \in$ $\underline{\boldsymbol{R}}$. Then $\underline{u} \in \underline{\boldsymbol{R}}$ and $\dot{n} \in \boldsymbol{G}$ be such that $\varphi * \dot{n} \in \underline{\boldsymbol{R}}$. As $\underline{\boldsymbol{R}}$ is a deductive system of $\boldsymbol{G}$, so it follows that $\dot{n} \in \underline{\boldsymbol{R}}$. Hence $\underline{\boldsymbol{R}}$ is a deductive system of $\boldsymbol{W}$.

(ii) We need to show that $\boldsymbol{G} / \underline{\boldsymbol{R}} \odot \boldsymbol{W} / \underline{\boldsymbol{R}} \subseteq W / \underline{\boldsymbol{R}}$ and $\boldsymbol{W} / \underline{\boldsymbol{R}} \odot \boldsymbol{G} / \underline{\boldsymbol{R}} \subseteq \boldsymbol{W} / \underline{\boldsymbol{R}}$ and $\forall \underline{\boldsymbol{R}}^{\dot{n}}$ $\in \boldsymbol{W} / \underline{\boldsymbol{R}}$ and $\underline{\boldsymbol{R}}^{r} \in \boldsymbol{G} / \underline{\boldsymbol{R}} \ni \underline{\boldsymbol{R}}^{\dot{n}} \circledast \underline{\boldsymbol{R}}^{r} \in \boldsymbol{W} / \underline{\boldsymbol{R}}$ implies that $\underline{\boldsymbol{R}}^{r} \in \boldsymbol{W} / \underline{\boldsymbol{R}}$. Let $\underline{\boldsymbol{R}}^{r} \odot \underline{\boldsymbol{R}}^{\hat{e}} \in$ $\boldsymbol{G} / \underline{\boldsymbol{R}} \odot \boldsymbol{W} / \underline{\boldsymbol{R}}$, then $\underline{\boldsymbol{R}}^{r} \odot{ }^{\hat{e}} \in \boldsymbol{G} / \underline{\boldsymbol{R}} \odot \boldsymbol{W} / \underline{\boldsymbol{R}}$. Now since $v \odot \hat{e} \in \boldsymbol{G} \odot \boldsymbol{W}$ and $\boldsymbol{G} \odot \boldsymbol{W} \subseteq \boldsymbol{W}$ so, we get $v \odot \hat{e} \in \boldsymbol{W}$. From here we have $\underline{\boldsymbol{R}}^{v} \odot{ }^{\hat{e}} \in \boldsymbol{W} / \underline{\boldsymbol{R}} \Rightarrow \underline{\boldsymbol{R}}^{v} \odot \underline{\boldsymbol{R}}^{\hat{e}} \in \boldsymbol{W} / \underline{\boldsymbol{R}} \Rightarrow \boldsymbol{G} / \underline{\boldsymbol{R}} \odot \boldsymbol{W} / \underline{\boldsymbol{R}}$ $\subseteq \boldsymbol{W} / \underline{\boldsymbol{R}}$. Now let $\underline{\boldsymbol{R}}^{\hat{e}} \odot \underline{\boldsymbol{R}}^{v} \in \boldsymbol{G} / \underline{\boldsymbol{R}} \odot \boldsymbol{W} / \underline{\boldsymbol{R}}$, then $\underline{\boldsymbol{R}}^{\hat{e}} \odot{ }^{r} \in \boldsymbol{W} / \underline{\boldsymbol{R}} \odot \boldsymbol{G} / \underline{\boldsymbol{R}}$. Now as $\hat{e} \odot v \in$ $\boldsymbol{W} \odot \boldsymbol{G}$ and $\boldsymbol{W} \odot \boldsymbol{G} \subseteq \boldsymbol{W}$ so, $\hat{e} \odot v \in \boldsymbol{W}$. It follows that $\underline{\boldsymbol{R}}^{\hat{e}} \odot{ }^{r} \in \boldsymbol{W} / \underline{\boldsymbol{R}} \Rightarrow \underline{\boldsymbol{R}}^{\hat{e}} \odot \underline{\boldsymbol{R}}^{v} \in \boldsymbol{W} / \underline{\boldsymbol{R}}$. It again implies that $\boldsymbol{W} / \underline{\boldsymbol{R}} \odot \boldsymbol{G} / \underline{\boldsymbol{R}} \subseteq \boldsymbol{W} / \underline{\boldsymbol{R}}$. Now for the second axiom of deductive systems, let $\underline{\boldsymbol{R}}^{v} \in \boldsymbol{G} / \underline{\boldsymbol{R}}$ and $\underline{\boldsymbol{R}}^{\dot{n}} \in \boldsymbol{W} / \underline{\boldsymbol{R}}$ be such that $\underline{\boldsymbol{R}}^{\dot{n}} \circledast \underline{\boldsymbol{R}}^{v} \in \boldsymbol{W} / \underline{\boldsymbol{R}}$ which implies that, $\underline{\boldsymbol{R}}^{\dot{n}} *^{r}$ $\in \boldsymbol{W} / \underline{\boldsymbol{R}} \Rightarrow \dot{n} * v \in \boldsymbol{W} \Rightarrow v \in \boldsymbol{W}$. It follows that $\underline{\boldsymbol{R}}^{v} \in \boldsymbol{W} / \underline{\boldsymbol{R}}$. Hence $\boldsymbol{W} / \underline{\boldsymbol{R}}$ is a deductive system of $\boldsymbol{G} / \underline{R}$.

\section{CONCLUSION}

In this paper, we have defined deductive systems in generalized BE-semigroups. Various properties of these structures have been investigated. Some non-trivial examples have been constructed to establish their existence. Congruence relations have been defined with the help of these systems which have further been used to define factor generalized BE-semigroups. 
The results presented will be of interest to researchers in various fields of science including mathematics and computer science.

\section{REFERENCES}

[1] K. Is'eki and S. Tanaka, "An introduction to the theory of BCK-algebras", Mathematica Japonica, 23 (1), 1-26 (1978).

[2] K. Is'eki, “On BCI-algebras”, Mathematics Seminar Notes, 8 (1), 125-130 (1980).

[3] Q. P. Hu and X. Li, “On BCH-algebras”, Mathematics Seminar Notes, 11 (2), 313-320 (1983).

[4] Q. P. Hu and X. Li, "On proper BCH-algebras", Mathematica Japonica, 30 (4), 659-661 (1985).

[5] J. Meng and Y.B. Jun, BCK-algebras, Kyung Moon Sa, Seoul, Korea, (1984).

[6] J. Neggers and H.S. Kim, “On d-algebras”, Mathematica Slovaca, 49, 19-26 (1999).

[7] J. Neggers, and H. S. Kim, “On B-algebras”, Matematichki Vesnik, 54 (1-2), $21-29$ (2002).

[8] J. Neggers and H. S. Kim, "A fundamental theorem of B-homomorphism for B-algebras", International Mathematical Journal, 2 (3), 207-214 (2002).

[9] Y.B. Jun, E.H. Roh and H.S. Kim, "On BH-algebras", Scientiae Mathematicae Japonicae Online, 1, 347-354 (1998).

[10] A. Walendziak, "Some axiomatizations of B-algebras", Mathematica Slovaca, 56 (3), 301-306 (2006).

[11] H. S. Kim, Y. H. Kim and J. Neggers, "Coxeter algebras and pre-Coxeter algebras in Smarandache setting", Honam Mathematical Journal, 26 (4), 471-481 (2004).

[12] C. B. Kim and H. S. Kim, "On BM-algebras", Scientiae Mathematicae Japonicae, 63 (3), 421-427 (2006).

[13] H.S. Kim and Y. H. Kim, "On BE-algebras", Scientiae Mathematicae Japonicae Online, 66 (1), 113-116 (2007).

[14] S.S. Ahn and K.S. So, "On ideals and upper sets in BE-algebras", Scientiae Mathematicae Japonicae, 68 (2), 279-285 (2008).

[15] S.S. Ahn and K.S. So, "On generalized upper sets in BE-algebras", Bulletin of the Korean Mathematical Society, 46 (2), 281-287 (2009).

[16] S.S. Ahn and Y.H. Kim, “On BE-semigroups", International Journal of Mathematics and Mathematical Sciences, 2011, Article ID 676020, 8 pages (2011).

[17] P. Yiarayong and P. Wachirawongsakorn, "A new generalization of BE-algebras", Heliyon, 4, Article ID e00863, 17 pages (2018).

[18] Javeria, Generalized BE-semigroups, M.Phil. Thesis, Abbottabad University of Science and Technology, Abbottabad, Pakistan, (2019).

Received February 18, 2020 